\title{
GENERALIZED ANALYTIC FEYNMAN INTEGRAL VIA FUNCTION SPACE INTEGRAL OF BOUNDED CYLINDER FUNCTIONALS
}

\author{
Seung Jun Chang, Jae Gil Choi, and Hyun Soo Chung
}

\begin{abstract}
In this paper, we use a generalized Brownian motion to define a generalized analytic Feynman integral. We then obtain some results for the generalized analytic Feynman integral of bounded cylinder functionals of the form

$$
F(x)=\hat{\nu}\left(\left(g_{1}, x\right)^{\sim}, \ldots,\left(g_{n}, x\right)^{\sim}\right)
$$

defined on a very general function space $C_{a, b}[0, T]$. We also present a change of scale formula for function space integrals of such cylinder functionals.
\end{abstract}

\section{Introduction}

In 1987, Cameron and Storvick expressed the analytic Feynman integral as the limit of a sequence of Wiener integrals and derived a change of scale formula for Wiener integrals of bounded functionals in a Banach algebra $\mathcal{S}$ on classical Wiener space, see [2] and [3]. Various kinds of those studies for Wiener integrals of bounded and unbounded functionals were developed on Yeh-Wiener space [16, 21], abstract Wiener space [12], [17], [18], [19], [20] and space of abstract Wiener space valued continuous functions on compact interval in $\mathbb{R}[10,11]$.

In this paper, we establish a relationship between the generalized analytic Feynman integral and the function space integral for bounded cylinder functionals of the form

$$
F(x)=\hat{\nu}\left(\left(g_{1}, x\right)^{\sim}, \ldots,\left(g_{n}, x\right)^{\sim}\right),
$$

where $\hat{\nu}: \mathbb{R}^{n} \rightarrow \mathbb{C}$ is the Fourier transform of complex-valued Borel measure $\nu$ on $\mathcal{B}\left(\mathbb{R}^{n}\right)$ and $(g, x)^{\sim}$ denotes the Paley-Wiener-Zygmund stochastic integral, but with $x$ in a very general function space $C_{a, b}[0, T]$ rather than in classical Wiener space. We then present a change of scale formula for function space integrals of such cylinder functionals.

Received August 1, 2009; Revised September 23, 2009.

2010 Mathematics Subject Classification. 60J65, 28C20.

Key words and phrases. generalized Brownian motion, generalized analytic Feynman integral, function space integral, cylinder functional, change of scale formula. 
The function space $C_{a, b}[0, T]$ induced by generalized Brownian motion was introduced by J. Yeh in [14] and was used extensively by Chang and Chung [6] and Chang and Skoug [9]. The Wiener process used in [2], [3], [10], [11], $[12],[16],[17],[18],[19],[20],[21]$ is free of drift and stationary in time while the stochastic process used in this paper, as well as in [6], [7], [8], [9], [14], is nonstationary in time and is subject to a drift $a(t)$. It turns out, as noted in Remark 3.5 below, that including a drift term $a(t)$ makes establishing the existence of generalized analytic Feynman integrals of functionals on $C_{a, b}[0, T]$ very difficult.

\section{Definitions and preliminaries}

In this section, we briefly list some of the preliminaries from [7], [8], [9] that we need to establish our results in next section; for more details, see [7], [8], $[9]$.

Let $\left(C_{a, b}[0, T], \mathcal{B}\left(C_{a, b}[0, T]\right), \mu\right)$ denote the function space induced by the generalized Brownian motion process $Y$ determined by $a(t)$ and $b(t)$ where $\mathcal{B}\left(C_{a, b}[0, T]\right)$ is the Borel $\sigma$-algebra induced by sup-norm, see [14] and [15, Chapters 3 and 4]. We assume in this paper that $a(t)$ is an absolutely continuous real-valued function on $[0, T]$ with $a(0)=0, a^{\prime}(t) \in L^{2}[0, T]$, and $b(t)$ is a strictly increasing, continuously differentiable real-valued function with $b(0)=0$ and $b^{\prime}(t)>0$ for each $t \in[0, T]$. Then we can consider the coordinate process $X:[0, T] \times C_{a, b}[0, T] \rightarrow \mathbb{R}$ given by $X(t, x)=x(t)$ which is a continuous version of $Y$ [15, Theorem 14.2]. Thus, for any $t \in[0, T]$ and $x \in C_{a, b}[0, T]$ we have

$$
X(t, x)=x(t) \sim N(a(t), b(t)) .
$$

A subset $B$ of $C_{a, b}[0, T]$ is said to be scale-invariant measurable provided $\rho B$ is $\mu$-measurable for all $\rho>0$, and a scale-invariant measurable set $N$ is said to be scale-invariant null provided $\mu(\rho N)=0$ for all $\rho>0$. A property that holds except on a scale-invariant null set is said to hold scale-invariant almost everywhere (s-a.e.).

Let $L_{a, b}^{2}[0, T]$ be the set of functions on $[0, T]$ which are Lebesgue measurable and square integrable with respect to the Lebesgue-Stieltjes measures on $[0, T]$ induced by $a(\cdot)$ and $b(\cdot)$; i.e.,

$$
L_{a, b}^{2}[0, T]=\left\{v: \int_{0}^{T} v^{2}(s) d b(s)<\infty \text { and } \int_{0}^{T} v^{2}(s) d|a|(s)<\infty\right\},
$$

where $|a|(t)$ denotes the total variation of the function $a(\cdot)$ on the interval $[0, t]$.

For $u, v \in L_{a, b}^{2}[0, T]$, let

$$
(u, v)_{a, b}=\int_{0}^{T} u(t) v(t) d[b(t)+|a|(t)] .
$$

Then $(\cdot, \cdot)_{a, b}$ is an inner product on $L_{a, b}^{2}[0, T]$ and $\|u\|_{a, b}=\sqrt{(u, u)_{a, b}}$ is a norm on $L_{a, b}^{2}[0, T]$. In particular, note that $\|u\|_{a, b}=0$ if and only if $u(t)=0$ for 
$m_{L}$-a.e. on $[0, T]$, where $m_{L}$ is the Lebesgue measure on $[0, T]$. Furthermore, $\left(L_{a, b}^{2}[0, T],\|\cdot\|_{a, b}\right)$ is a separable Hilbert space.

Let $\left\{\phi_{j}\right\}_{j=1}^{\infty}$ be a complete orthonormal set of real-valued functions of bounded variation on $[0, T]$ such that

$$
\left(\phi_{j}, \phi_{k}\right)_{a, b}= \begin{cases}0, & j \neq k \\ 1, & j=k\end{cases}
$$

Then for each $v \in L_{a, b}^{2}[0, T]$, the Paley-Wiener-Zygmund (PWZ) stochastic integral $\langle v, x\rangle$ is defined by the formula

$$
\langle v, x\rangle=\lim _{n \rightarrow \infty} \int_{0}^{T} \sum_{j=1}^{n}\left(v, \phi_{j}\right)_{a, b} \phi_{j}(t) d x(t)
$$

for all $x \in C_{a, b}[0, T]$ for which the limit exists; one can show that for each $v \in L_{a, b}^{2}[0, T]$, the PWZ stochastic integral $\langle v, x\rangle$ exists for $\mu$-a.e. $x \in C_{a, b}[0, T]$ and if $v$ is of bounded variation on $[0, T]$, then the PWZ stochastic integral $\langle v, x\rangle$ equals the Riemann-Stieltjes integral $\int_{0}^{T} v(t) d x(t)$ for s-a.e. $x \in C_{a, b}[0, T]$. For more details, see [9].

Let

$$
C_{a, b}^{\prime}[0, T]=\left\{w \in C_{a, b}[0, T]: w(t)=\int_{0}^{t} z(s) d b(s) \text { for some } z \in L_{a, b}^{2}[0, T]\right\} .
$$

For $w \in C_{a, b}^{\prime}[0, T]$, with $w(t)=\int_{0}^{t} z(s) d b(s)$ for $t \in[0, T]$, let $D_{t}: C_{a, b}^{\prime}[0, T] \rightarrow$ $L_{a, b}^{2}[0, T]$ be defined by the formula

$$
D_{t} w=z(t)=\frac{w^{\prime}(t)}{b^{\prime}(t)} \text {. }
$$

Then $C_{a, b}^{\prime}[0, T]$ with inner product

$$
\left(w_{1}, w_{2}\right)_{C_{a, b}^{\prime}}=\int_{0}^{T} D_{t} w_{1} D_{t} w_{2} d b(t)
$$

is a separable Hilbert space. Furthermore, $\left(C_{a, b}^{\prime}[0, T], C_{a, b}[0, T], \mu\right)$ is an example of abstract Wiener space. Note that two separable Hilbert spaces $L_{a, b}^{2}[0, T]$ and $C_{a, b}^{\prime}[0, T]$ are homeomorphic.

Throughout this paper we assume $a \in C_{a, b}^{\prime}[0, T]$ and for $w \in C_{a, b}^{\prime}[0, T]$, with $w(t)=\int_{0}^{t} z(s) d b(s)$ for $t \in[0, T]$, we also use the notation $(w, x)^{\sim}$ instead of $\langle z, x\rangle=\left\langle D_{t} w, x\right\rangle$. Then we have the following assertions.

(1) For each $w \in C_{a, b}^{\prime}[0, T]$, the random variable $x \mapsto(w, x)^{\sim}$ is Gaussian with mean $(w, a)_{C_{a, b}^{\prime}}$ and variance $\|w\|_{C_{a, b}^{\prime}}^{2}$.

(2) $(w, \alpha x)^{\sim}=(\alpha w, x)^{\sim}=\alpha(w, x)^{\sim}$ for any real number $\alpha, w \in C_{a, b}^{\prime}[0, T]$ and $x \in C_{a, b}[0, T]$.

(3) If $\left\{w_{1}, w_{2}, \ldots, w_{n}\right\}$ is an orthogonal set in $C_{a, b}^{\prime}[0, T]$, then the random variables $\left(w_{i}, x\right)^{\sim}$ 's are independent. 
We denote the function space integral of a $\mathcal{B}\left(C_{a, b}[0, T]\right)$-measurable functional $F$ by

whenever the integral exists.

$$
E[F]=\int_{C_{a, b}[0, T]} F(x) d \mu(x)
$$

Throughout this paper, let $\mathbb{C}, \mathbb{C}_{+}$and $\tilde{\mathbb{C}}_{+}$denote the complex numbers, the complex numbers with positive real part, and the nonzero complex numbers with nonnegative real part, respectively.

We are now ready to state the definition of the generalized analytic Feynman integral.

Definition 2.1. Let $F: C_{a, b}[0, T] \rightarrow \mathbb{C}$ be such that the function space integral $J(\lambda)=E\left[F\left(\lambda^{-1 / 2} \cdot\right)\right]$ exists as a finite number for all $\lambda>0$. If there exists a function $J^{*}(\lambda)$ analytic in $\mathbb{C}_{+}$such that $J^{*}(\lambda)=J(\lambda)$ for all $\lambda>0$, then $J^{*}(\lambda)$ is defined to be the analytic function space integral of $F$ over $C_{a, b}[0, T]$ with parameter $\lambda$, and for $\lambda \in \mathbb{C}_{+}$we write

$$
E^{\operatorname{an}_{\lambda}}[F] \equiv E_{x}^{\operatorname{an}_{\lambda}}[F(x)]=J^{*}(\lambda) .
$$

Let $q \neq 0$ be a real number and let $F$ be a functional such that $E^{a_{\lambda}}[F]$ exists for all $\lambda \in \mathbb{C}_{+}$. If the following limit exists, we call it the generalized analytic Feynman integral of $F$ with parameter $q$ and we write

$$
E^{\operatorname{anf}_{q}}[F] \equiv E_{x}^{\operatorname{anf}_{q}}[F(x)]=\lim _{\lambda \rightarrow-i q} E^{\operatorname{an}_{\lambda}}[F]
$$

where $\lambda$ approaches $-i q$ through values in $\mathbb{C}_{+}$.

We will finish this section by mentioning the following useful formula for function space integral.

Theorem 2.2. Let $\left\{g_{1}, \ldots, g_{n}\right\}$ be an orthonormal set in $C_{a, b}^{\prime}[0, T]$ and let $F: C_{a, b}[0, T] \rightarrow \mathbb{C}$ be a measurable functional defined by

$$
F(x)=f\left(\left(g_{1}, x\right)^{\sim}, \ldots,\left(g_{n}, x\right)^{\sim}\right),
$$

where $f: \mathbb{R}^{n} \rightarrow \mathbb{C}$ is a Borel measurable function. Then

$$
\begin{aligned}
E[F] & \equiv \int_{C_{a, b}[0, T]} f\left(\left(g_{1}, x\right)^{\sim}, \ldots,\left(g_{n}, x\right)^{\sim}\right) d \mu(x) \\
& =(2 \pi)^{-n / 2} \int_{\mathbb{R}^{n}} f(\vec{u}) \exp \left\{-\frac{1}{2} \sum_{j=1}^{n}\left(u_{j}-\left(g_{j}, a\right)_{C_{a, b}^{\prime}}\right)^{2}\right\} d \vec{u}
\end{aligned}
$$

in the sense that if either side exists, both sides exist and equality holds.

To establish our results in next section, the following well-known integration formula is useful:

$$
\int_{\mathbb{R}} \exp \left\{-\alpha u^{2}+\beta u\right\} d u=\left(\frac{\pi}{\alpha}\right)^{\frac{1}{2}} \exp \left\{\frac{\beta^{2}}{4 \alpha}\right\}
$$

for all complex numbers $\alpha$ and $\beta$ with $\operatorname{Re}(\alpha)>0$. 
In this paper, for each $\lambda \in \widetilde{\mathbb{C}}_{+}, \lambda^{-1 / 2}$ (or $\lambda^{1 / 2}$ ) is always chosen to have positive real part.

\section{Generalized Feynman integral and change of scale formula of cylinder functionals}

In this section, we give a class of certain bounded cylinder functionals whose analytic function space integral and generalized analytic Feynman integral exist under appropriate conditions and we show that the generalized analytic Feynman integral of such cylinder functionals can be expressed as the limit of a sequence of function space integrals. Finally, we obtain a change of scale formula for function space integrals of cylinder functionals.

A functional $F$ is called a cylinder functional on $C_{a, b}[0, T]$ if there exists a finite subset $\left\{h_{1}, \ldots, h_{m}\right\}$ of $C_{a, b}^{\prime}[0, T]$ such that

$$
F(x)=\psi\left(\left(h_{1}, x\right)^{\sim}, \ldots,\left(h_{m}, x\right)^{\sim}\right), \quad x \in C_{a, b}[0, T],
$$

where $\psi$ is a complex-valued Borel measurable function on $\mathbb{R}^{m}$. It is easy to show that for given cylinder functional $F$ of the form (3.1) there exists an orthonormal subset $\left\{g_{1}, \ldots, g_{n}\right\}$ of $C_{a, b}^{\prime}[0, T]$ such that $F$ is expressed as

$$
F(x)=f\left(\left(g_{1}, x\right)^{\sim}, \ldots,\left(g_{n}, x\right)^{\sim}\right), \quad x \in C_{a, b}[0, T],
$$

where $f$ is a complex-valued Borel measurable function on $\mathbb{R}^{n}$. Thus we lose no generality in assuming that every cylinder functional on $C_{a, b}[0, T]$ is of the form (3.2).

Definition 3.1. Let $\mathcal{M}\left(\mathbb{R}^{n}\right)$ denote the space of complex-valued Borel measures on $\mathcal{B}\left(\mathbb{R}^{n}\right)$. It is well known that a complex-valued Borel measure $\nu$ necessarily has a finite total variation $\|\nu\|$, and $\mathcal{M}\left(\mathbb{R}^{n}\right)$ is a Banach algebra under the norm $\|\cdot\|$ and with convolution as multiplication.

For $\nu \in \mathcal{M}\left(\mathbb{R}^{n}\right)$, the Fourier transform $\hat{\nu}$ of $\nu$ is a complex-valued function defined on $\mathbb{R}^{n}$ by the formula

$$
\hat{\nu}(\vec{u})=\int_{\mathbb{R}^{n}} \exp \left\{i \sum_{j=1}^{n} u_{j} v_{j}\right\} d \nu(\vec{v}),
$$

where $\vec{u}=\left(u_{1}, \ldots, u_{n}\right)$ and $\vec{v}=\left(v_{1}, \ldots, v_{n}\right)$ are in $\mathbb{R}^{n}$.

Let $\left\{g_{1}, \ldots, g_{n}\right\}$ be an orthonormal subset of $C_{a, b}^{\prime}[0, T]$. Define the functional $F: C_{a, b}[0, T] \rightarrow \mathbb{C}$ by

$$
F(x)=\hat{\nu}\left(\left(g_{1}, x\right)^{\sim}, \ldots,\left(g_{n}, x\right)^{\sim}\right), \quad x \in C_{a, b}[0, T],
$$

where $\hat{\nu}$ is the Fourier transform of $\nu$ in $\mathcal{M}\left(\mathbb{R}^{n}\right)$. Then $F$ is a bounded cylinder functional and $|\hat{\nu}(\vec{u})| \leq\|\nu\|<+\infty$. Throughout this paper, we fix the positive integer $n$.

We first show that the analytic function space integral of the functional $F$ given by the equation (3.4) exists. 
Theorem 3.2. Let $F$ be given by the equation (3.4). Then for each $\lambda \in \mathbb{C}_{+}$, the analytic function space integral $E^{\mathrm{an}_{\lambda}}[F]$ exists and is given by the formula

$$
E^{\operatorname{an}_{\lambda}}[F]=\int_{\mathbb{R}^{n}} \exp \left\{-\frac{1}{2 \lambda} \sum_{j=1}^{n} v_{j}^{2}+i \lambda^{-1 / 2} \sum_{j=1}^{n}\left(g_{j}, a\right)_{C_{a, b}^{\prime}} v_{j}\right\} d \nu(\vec{v}) .
$$

Proof. By (3.4), (3.3), the Fubini theorem, (2.1) and (2.2), we have that for all $\lambda>0$,

$$
\begin{aligned}
J(\lambda) & =E_{x}\left[F\left(\lambda^{-1 / 2} x\right)\right] \\
& =\int_{\mathbb{R}^{n}} E_{x}\left[\exp \left\{i \lambda^{-1 / 2} \sum_{j=1}^{n}\left(g_{j}, x\right)^{\sim} v_{j}\right\}\right] d \nu(\vec{v}) \\
& =(2 \pi)^{-n / 2} \int_{\mathbb{R}^{n}} \int_{\mathbb{R}^{n}} \exp \left\{i \lambda^{-1 / 2} \sum_{j=1}^{n} u_{j} v_{j}\right. \\
& =\int_{\mathbb{R}^{n}} \exp \left\{-\frac{1}{2} \sum_{j=1}^{n}\left(u_{j}-\left(g_{j}, a\right)_{C_{a, b}^{\prime}}\right)^{2}\right\} d \vec{u} d \nu(\vec{v})
\end{aligned}
$$

Now let

$$
J^{*}(\lambda)=\int_{\mathbb{R}^{n}} \exp \left\{-\frac{1}{2 \lambda} \sum_{j=1}^{n} v_{j}^{2}+i \lambda^{-1 / 2} \sum_{j=1}^{n}\left(g_{j}, a\right)_{C_{a, b}^{\prime}} v_{j}\right\} d \nu(\vec{v})
$$

for $\lambda \in \mathbb{C}_{+}$. Then $J^{*}(\lambda)=J(\lambda)$ for all $\lambda>0$. We will use the Morera theorem to show that $J^{*}(\lambda)$ is analytic on $\mathbb{C}_{+}$. Let $\left\{\lambda_{l}\right\}_{l=1}^{\infty}$ be a sequence in $\mathbb{C}_{+}$such that $\lambda_{l} \rightarrow \lambda$ through $\mathbb{C}_{+}$. Then $\lambda_{l}^{-1 / 2} \rightarrow \lambda^{-1 / 2}$ and $\operatorname{Re}\left(\lambda_{l}\right) \neq 0$ for all $l \in \mathbb{N}$. Thus we have that for each $l \in \mathbb{N}$,

$$
\begin{aligned}
& \left|\exp \left\{-\frac{1}{2 \lambda_{l}} \sum_{j=1}^{n} v_{j}^{2}+i \lambda_{l}^{-1 / 2} \sum_{j=1}^{n}\left(g_{j}, a\right)_{C_{a, b}^{\prime}} v_{j}\right\}\right| \\
& =\exp \left\{-\frac{\operatorname{Re}\left(\lambda_{l}\right)}{2\left|\lambda_{l}\right|^{2}} \sum_{j=1}^{n} v_{j}^{2}-\operatorname{Im}\left(\lambda_{l}^{-1 / 2}\right) \sum_{j=1}^{n}\left(g_{j}, a\right)_{C_{a, b}^{\prime}} v_{j}\right\} \\
& =\exp \left\{-\frac{1}{2} \sum_{j=1}^{n}\left(\frac{\sqrt{\operatorname{Re}\left(\lambda_{l}\right)}}{\left|\lambda_{l}\right|} v_{j}+\frac{\left|\lambda_{l}\right| \operatorname{Im}\left(\lambda_{l}^{-1 / 2}\right)}{\sqrt{\operatorname{Re}\left(\lambda_{l}\right)}}\left(g_{j}, a\right)_{C_{a, b}^{\prime}}\right)^{2}\right. \\
& \left.+\frac{1}{2} \sum_{j=1}^{n} \frac{\left|\lambda_{l}\right|^{2}\left(\operatorname{Im}\left(\lambda_{l}^{-1 / 2}\right)\right)^{2}}{\operatorname{Re}\left(\lambda_{l}\right)}\left(g_{j}, a\right)_{C_{a, b}^{\prime}}^{2}\right\} \\
& \leq \exp \left\{\frac{\left|\lambda_{l}\right|^{2}\left(\operatorname{Im}\left(\lambda_{l}^{-1 / 2}\right)\right)^{2}}{2 \operatorname{Re}\left(\lambda_{l}\right)} \sum_{j=1}^{n}\left(g_{j}, a\right)_{C_{a, b}^{\prime}}^{2}\right\} .
\end{aligned}
$$


Since $\nu \in \mathcal{M}\left(\mathbb{R}^{n}\right)$, we see that

$$
\begin{array}{r}
\left|\int_{\mathbb{R}^{n}} \exp \left\{\frac{\left|\lambda_{l}\right|^{2}\left(\operatorname{Im}\left(\lambda_{l}^{-1 / 2}\right)\right)^{2}}{2 \operatorname{Re}\left(\lambda_{l}\right)} \sum_{j=1}^{n}\left(g_{j}, a\right)_{C_{a, b}^{\prime}}^{2}\right\} d \nu(\vec{v})\right| \\
\leq \exp \left\{\frac{\left|\lambda_{l}\right|^{2}\left(\operatorname{Im}\left(\lambda_{l}^{-1 / 2}\right)\right)^{2}}{2 \operatorname{Re}\left(\lambda_{l}\right)} \sum_{j=1}^{n}\left(g_{j}, a\right)_{C_{a, b}^{\prime}}^{2}\right\}\|\nu\|<+\infty
\end{array}
$$

for each $l \in \mathbb{N}$. Furthermore we have that

$$
\begin{aligned}
& \lim _{l \rightarrow \infty} \int_{\mathbb{R}^{n}} \exp \left\{\frac{\left|\lambda_{l}\right|^{2}\left(\operatorname{Im}\left(\lambda_{l}^{-1 / 2}\right)\right)^{2}}{2 \operatorname{Re}\left(\lambda_{l}\right)} \sum_{j=1}^{n}\left(g_{j}, a\right)_{C_{a, b}^{\prime}}^{2}\right\} d \nu(\vec{v}) \\
= & \lim _{l \rightarrow \infty} \exp \left\{\frac{\left|\lambda_{l}\right|^{2}\left(\operatorname{Im}\left(\lambda_{l}^{-1 / 2}\right)\right)^{2}}{2 \operatorname{Re}\left(\lambda_{l}\right)} \sum_{j=1}^{n}\left(g_{j}, a\right)_{C_{a, b}^{\prime}}^{2}\right\} \nu\left(\mathbb{R}^{n}\right) \\
= & \exp \left\{\frac{|\lambda|^{2}\left(\operatorname{Im}\left(\lambda^{-1 / 2}\right)\right)^{2}}{2 \operatorname{Re}(\lambda)} \sum_{j=1}^{n}\left(g_{j}, a\right)_{C_{a, b}^{\prime}}^{2}\right\} \nu\left(\mathbb{R}^{n}\right) \\
= & \int_{\mathbb{R}^{n}} \exp \left\{\frac{|\lambda|^{2}\left(\operatorname{Im}\left(\lambda^{-1 / 2}\right)\right)^{2}}{2 \operatorname{Re}(\lambda)} \sum_{j=1}^{n}\left(g_{j}, a\right)_{C_{a, b}^{\prime}}^{2}\right\} d \nu(\vec{v}) .
\end{aligned}
$$

Thus, by Theorem 4.17 in $[13$, p. 92$], J^{*}(\lambda)$ is continuous on $\mathbb{C}_{+}$. Since

$$
k(\lambda) \equiv \exp \left\{-\frac{1}{2 \lambda} \sum_{j=1}^{n} v_{j}^{2}+i \lambda^{-1 / 2} \sum_{j=1}^{n}\left(g_{j}, a\right)_{C_{a, b}^{\prime}} v_{j}\right\}
$$

is analytic on $\mathbb{C}_{+}$, applying the Fubini theorem, we have

$$
\int_{\triangle} J^{*}(\lambda) d \lambda=\int_{\mathbb{R}^{n}} \int_{\triangle} k(\lambda) d \lambda d \nu(\vec{v})=0
$$

for all rectifiable simple closed curve $\triangle$ lying in $\mathbb{C}_{+}$. Thus by the Morera theorem, $J^{*}(\lambda)$ is analytic on $\mathbb{C}_{+}$. Therefore the analytic function space integral $J^{*}(\lambda)=E^{\operatorname{an}_{\lambda}}[F]$ exists and is given by the equation (3.5).

Throughout this section, for convenience, we use the following notation:

$$
G(\lambda, x)=\exp \left\{\frac{1-\lambda}{2} \sum_{j=1}^{n}\left[\left(g_{j}, x\right)^{\sim}\right]^{2}+\left(\lambda^{1 / 2}-1\right) \sum_{j=1}^{n}\left(g_{j}, a\right)_{C_{a, b}^{\prime}}\left(g_{j}, x\right)^{\sim}\right\}
$$

for $\lambda \in \mathbb{C}_{+}$and $x \in C_{a, b}[0, T]$.

Theorem 3.3. Let $F$ be as in Theorem 3.2. Then for each $\lambda \in \mathbb{C}_{+}$,

(1) the function space integral $E[G(\lambda, \cdot) F]$ exists where $G$ is given by the equation (3.7) above, and

(2) the analytic function space integral $E^{a_{\lambda}}[F]$ of $F$ is given by the formula

$$
E^{\mathrm{an}_{\lambda}}[F]=\lambda^{n / 2} E[G(\lambda, \cdot) F] .
$$


Remark 3.4. Note that the equation (3.8) is indeed a useful formula for expressing the analytic function space integral in terms of ordinary function space integrals.

Proof of Theorem 3.3. By (3.4), (3.7), the Fubini theorem, (2.1) and (2.2), we have that for every $\lambda \in \mathbb{C}_{+}$

$$
\begin{aligned}
& E_{x}[G(\lambda, x) F(x)] \\
&=(2 \pi)^{-n / 2} \int_{\mathbb{R}^{n}} \int_{\mathbb{R}^{n}} \exp \left\{-\frac{\lambda}{2} \sum_{j=1}^{n} u_{j}^{2}+\sum_{j=1}^{n}\left(\lambda^{1 / 2}\left(g_{j}, a\right)_{C_{a, b}^{\prime}}+i v_{j}\right) u_{j}\right. \\
&\left.-\frac{1}{2} \sum_{j=1}^{n}\left(g_{j}, a\right)_{C_{a, b}^{\prime}}^{2}\right\} d \vec{u} d \nu(\vec{v}) \\
&=\lambda^{-n / 2} \int_{\mathbb{R}^{n}} \exp \left\{-\frac{1}{2 \lambda} \sum_{j=1}^{n} v_{j}^{2}+i \lambda^{-1 / 2} \sum_{j=1}^{n}\left(g_{j}, a\right)_{C_{a, b}^{\prime}} v_{j}\right\} d \nu(\vec{v}) .
\end{aligned}
$$

Using (3.6) with $\lambda_{l}$ replaced with $\lambda$, we see that the absolute value of the last expression of (3.9) is less than

$$
|\lambda|^{-n / 2} \exp \left\{\frac{|\lambda|^{2}\left(\operatorname{Im}\left(\lambda^{-1 / 2}\right)\right)^{2}}{2 \operatorname{Re}(\lambda)} \sum_{j=1}^{n}\left(g_{j}, a\right)_{C_{a, b}^{\prime}}^{2}\right\}\|\nu\|,
$$

and so the function space integral $E_{x}[G(\lambda, x) F(x)]$ exists. The equation (3.8) now follows from equations (3.5) and (3.9).

The observation below will be very useful in the development of our results for the generalized analytic Feynman integral of functionals given by the equation (3.4).

Remark 3.5. If $a(t) \equiv 0$ on $[0, T]$, then for all $F$ given by the equation (3.4), the generalized analytic Feynman integral $E^{\operatorname{anf}_{q}}[F]$ will always exist for all real $q \neq 0$ and be given by the formula

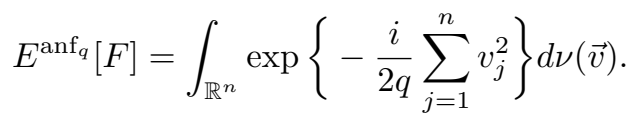

However for $a(t)$ as in Section 2, and proceeding formally using equations (3.4), (2.1) and (2.2), we see that $E^{\operatorname{anf}_{q}}[F]$ will be given by the equation (3.11) below if it exists. But the integral on the right-hand side of (3.11) might not exist if the real part of

$$
\left\{-\frac{i}{2 q} \sum_{j=1}^{n} v_{j}^{2}+i(-i q)^{-1 / 2} \sum_{j=1}^{n}\left(g_{j}, a\right)_{C_{a, b}^{\prime}} v_{j}\right\}
$$


is positive. However

$$
\begin{aligned}
& \left|\exp \left\{-\frac{i}{2 q} \sum_{j=1}^{n} v_{j}^{2}+i(-i q)^{-1 / 2} \sum_{j=1}^{n}\left(g_{j}, a\right)_{C_{a, b}^{\prime}} v_{j}\right\}\right| \\
& =\left\{\exp \left\{-(2 q)^{-1 / 2} \sum_{j=1}^{n}\left(g_{j}, a\right)_{C_{a, b}^{\prime}} v_{j}\right\}, \quad q>0\right. \\
& \exp \left\{(-2 q)^{-1 / 2} \sum_{j=1}^{n}\left(g_{j}, a\right)_{C_{a, b}^{\prime}} v_{j}\right\}, \quad q<0
\end{aligned}
$$

and so the generalized analytic Feynman integral $E^{\operatorname{anf}_{q}}[F]$ will certainly exist provided the associated measure $\nu$ of $F$ satisfies the condition

$$
\int_{\mathbb{R}^{n}} \exp \left\{\frac{\|a\|_{C_{a, b}^{\prime}}}{\sqrt{|2 q|}} \sum_{j=1}^{n}\left|v_{j}\right|\right\}|d \nu(\vec{v})|<+\infty .
$$

Note that in case $a(t) \equiv 0$ and $b(t)=t$ on $[0, T]$, the function space reduces to the classical Wiener space $C_{0}[0, T]$ and $\left(g_{j}, a\right)_{C_{a, b}^{\prime}}=0$ for all $j=1, \ldots, n$. Hence for all $\lambda \in \tilde{\mathbb{C}}_{+}$,

$$
\begin{aligned}
& \left|\exp \left\{-\frac{1}{2 \lambda} \sum_{j=1}^{n} v_{j}^{2}+i \lambda^{-1 / 2} \sum_{j=1}^{n}\left(g_{j}, a\right)_{C_{a, b}^{\prime}} v_{j}\right\}\right| \\
= & \left|\exp \left\{-\frac{1}{2 \lambda} \sum_{j=1}^{n} v_{j}^{2}\right\}\right|=\exp \left\{-\frac{\operatorname{Re}(\lambda)}{2|\lambda|^{2}} \sum_{j=1}^{n} v_{j}^{2}\right\} \leq 1 .
\end{aligned}
$$

Theorem 3.6. Let $F$ be as in Theorem 3.2. Let $q_{0}$ be a nonzero real number. Suppose that the associated measure $\nu$ of $F$ satisfies condition (3.10) with $q$ replaced with $q_{0}$. Then, for all real $q$ with $|q|>\left|q_{0}\right|$, the generalized analytic Feynman integral $E^{\operatorname{anf}_{q}}[F]$ exists and is given by the formula

$$
E^{\operatorname{anf}_{q}}[F]=\int_{\mathbb{R}^{n}} \exp \left\{-\frac{i}{2 q} \sum_{j=1}^{n} v_{j}^{2}+i(-i q)^{-1 / 2} \sum_{j=1}^{n}\left(g_{j}, a\right)_{C_{a, b}^{\prime}} v_{j}\right\} d \nu(\vec{v}) .
$$

Proof. Let $\left\{\lambda_{l}\right\}_{l=1}^{\infty}$ be a sequence of complex numbers such that $\lambda_{l} \rightarrow-i q$ through $\mathbb{C}_{+}$and for each $l \in \mathbb{N}$, let

$$
f_{l}(\vec{v})=\exp \left\{-\left(1 / 2 \lambda_{l}\right) \sum_{j=1}^{n} v_{j}^{2}+i \lambda_{l}^{-1 / 2} \sum_{j=1}^{n}\left(g_{j}, a\right)_{C_{a, b}^{\prime}} v_{j}\right\} .
$$

Then $f_{l}(\vec{v})$ converges to

$$
f(\vec{v}) \equiv \exp \left\{-(i / 2 q) \sum_{j=1}^{n} v_{j}^{2}+i(-i q)^{-1 / 2} \sum_{j=1}^{n}\left(g_{j}, a\right)_{C_{a, b}^{\prime}} v_{j}\right\} .
$$


By Theorem 3.2, for all $l \in \mathbb{N}, \int_{\mathbb{R}^{n}} f_{l}(\vec{v}) d \nu(\vec{v})$ exists. Since $\left|\arg \left(\lambda_{l}^{-1 / 2}\right)\right|<\pi / 4$ for every $l \in \mathbb{N}$ and $\lambda_{l}^{-1 / 2}=\operatorname{Re}\left(\lambda_{l}^{-1 / 2}\right)+i \operatorname{Im}\left(\lambda_{l}^{-1 / 2}\right) \rightarrow(-i q)^{-1 / 2}=1 / \sqrt{|2 q|}+$ $i \operatorname{sign}(q) / \sqrt{|2 q|}$, we see that $\operatorname{Re}\left(\lambda_{l}^{-1 / 2}\right)>\left|\operatorname{Im}\left(\lambda_{l}^{-1 / 2}\right)\right|$ for every $l \in \mathbb{N}$ and there exists a sufficiently large $k \in \mathbb{N}$ such that $\left|\operatorname{Im}\left(\lambda_{l}^{-1 / 2}\right)\right|<1 / \sqrt{\left|q_{0}\right|}$ for every $l \geq k$. Thus for each $l \geq k$,

$$
\begin{aligned}
&\left|f_{l}(\vec{v})\right|=\mid \exp \left\{-\frac{1}{2}\left(\left[\operatorname{Re}\left(\lambda_{l}^{-1 / 2}\right)\right]^{2}-\left[\operatorname{Im}\left(\lambda_{l}^{-1 / 2}\right)\right]^{2}\right.\right.\left.+2 i \operatorname{Re}\left(\lambda_{l}^{-1 / 2}\right) \operatorname{Im}\left(\lambda_{l}^{-1 / 2}\right)\right) \sum_{j=1}^{n} v_{j}^{2} \\
&\left.\quad+i\left(\operatorname{Re}\left(\lambda_{l}^{-1 / 2}\right)+i \operatorname{Im}\left(\lambda_{l}^{-1 / 2}\right)\right) \sum_{j=1}^{n}\left(g_{j}, a\right)_{C_{a, b}^{\prime}} v_{j}\right\} \mid \\
& \leq \exp \left\{-\operatorname{Im}\left(\lambda_{l}^{-1 / 2}\right) \sum_{j=1}^{n}\left(g_{j}, a\right)_{C_{a, b}^{\prime}} v_{j}\right\} \\
& \leq \exp \left\{\left|\operatorname{Im}\left(\lambda_{l}^{-1 / 2}\right)\right|\|a\|_{C_{a, b}^{\prime}} \sum_{j=1}^{n}\left|v_{j}\right|\right\} \\
&<\exp \left\{\frac{\|a\|_{C_{a, b}^{\prime}}}{\sqrt{\left|2 q_{0}\right|}} \sum_{j=1}^{n}\left|v_{j}\right|\right\}
\end{aligned}
$$

and so, by the condition (3.10) with $q$ replaced with $q_{0}$,

$$
\begin{aligned}
\left|\int_{\mathbb{R}^{n}} f_{l}(\vec{v}) d \nu(\vec{v})\right| & \leq \int_{\mathbb{R}^{n}}\left|f_{l}(\vec{v})\right||d \nu(\vec{v})| \\
& <\int_{\mathbb{R}^{n}} \exp \left\{\frac{\|a\|_{C_{a, b}^{\prime}}}{\sqrt{\left|2 q_{0}\right|}} \sum_{j=1}^{n}\left|v_{j}\right|\right\}|d \nu(\vec{v})|<+\infty .
\end{aligned}
$$

Also, by the condition (3.10) with $q$ replaced with $q_{0}$, we have

$$
\begin{aligned}
\left|\int_{\mathbb{R}^{n}} f(\vec{v}) d \nu(\vec{v})\right| & \leq \int_{\mathbb{R}^{n}} \exp \left\{\frac{\|a\|_{C_{a, b}^{\prime}}}{\sqrt{|2 q|}} \sum_{j=1}^{n}\left|v_{j}\right|\right\}|d \nu(\vec{v})| \\
& <\int_{\mathbb{R}^{n}} \exp \left\{\frac{\|a\|_{C_{a, b}^{\prime}}}{\sqrt{\left|2 q_{0}\right|}} \sum_{j=1}^{n}\left|v_{j}\right|\right\}|d \nu(\vec{v})|<+\infty .
\end{aligned}
$$

Hence by the dominated convergence theorem, we have the equation (3.11).

In our next theorem, we express the generalized analytic Feynman integral of $F$ as the limit of a sequence of function space integrals on $C_{a, b}[0, T]$.

Theorem 3.7. Let $F$ and $q_{0}$ be as in Theorem 3.6. Let $\left\{\lambda_{l}\right\}_{l=1}^{\infty}$ be a sequence of complex numbers such that $\lambda_{l} \rightarrow-i q$ through $\mathbb{C}_{+}$, where $q$ is a nonzero 
real number with $|q|>\left|q_{0}\right|$. Then the generalized analytic Feynman integral $E^{\operatorname{anf}_{q}}[F]$ of $F$ is expressed as follows:

$$
E^{\operatorname{anf}_{q}}[F]=\lim _{l \rightarrow \infty} \lambda_{l}^{n / 2} E\left[G\left(\lambda_{l}, \cdot\right) F\right]
$$

where $G$ is given by the equation (3.7) above.

Proof. We can obtain from (3.9) that for all $l \in \mathbb{N}$,

$$
\begin{aligned}
& \lambda_{l}^{n / 2} E_{x}\left[G\left(\lambda_{l}, x\right) F(x)\right] \\
= & \int_{\mathbb{R}^{n}} \exp \left\{-\frac{1}{2 \lambda_{l}} \sum_{j=1}^{n} v_{j}^{2}+i \lambda_{l}^{-1 / 2} \sum_{j=1}^{n}\left(g_{j}, a\right)_{C_{a, b}^{\prime}} v_{j}\right\} d \nu(\vec{v}) .
\end{aligned}
$$

Thus, using the dominated convergence theorem, we have

$$
\begin{aligned}
& \lim _{l \rightarrow \infty} \lambda_{l}^{n / 2} E_{x}\left[G\left(\lambda_{l}, x\right) F(x)\right] \\
= & \lim _{l \rightarrow \infty} \int_{\mathbb{R}^{n}} \exp \left\{-\frac{1}{2 \lambda_{l}} \sum_{j=1}^{n} v_{j}^{2}+i \lambda_{l}^{-1 / 2} \sum_{j=1}^{n}\left(g_{j}, a\right)_{C_{a, b}^{\prime}} v_{j}\right\} d \nu(\vec{v}) \\
= & \int_{\mathbb{R}^{n}} \exp \left\{-\frac{i}{2 q} \sum_{j=1}^{n} v_{j}^{2}+i(-i q)^{-1 / 2} \sum_{j=1}^{n}\left(g_{j}, a\right)_{C_{a, b}^{\prime}} v_{j}\right\} d \nu(\vec{v}) .
\end{aligned}
$$

The equation (3.12) follows from equations (3.11) and (3.13).

Finally, we obtain a change of scale formula for function space integrals of functionals $F$ given by the equation (3.4).

Theorem 3.8. Let $F$ be as in Theorem 3.2. Then, for any $\rho>0$,

$$
E[F(\rho \cdot)]=\rho^{-n} E\left[G\left(\rho^{-2}, \cdot\right) F\right],
$$

where $G$ is given by the equation (3.7) above.

Proof. Using the equation (3.8) with $\lambda$ replaced with $\rho^{-2}$, we have the desired result.

\section{Examples}

In this section we present various functionals to apply our results in previous section.

Let $S: C_{a, b}^{\prime}[0, T] \rightarrow C_{a, b}^{\prime}[0, T]$ be the linear operator defined by $S w(t)=$ $\int_{0}^{t} w(s) d b(s)$. Then the adjoint operator $S^{*}$ of $S$ is given by

$$
S^{*} w(t)=\int_{0}^{t}(w(T)-w(s)) d b(s)
$$

It is easily shown that $S$ is injective. Let

$$
\psi(t)=\sqrt{3} b(T)^{-3 / 2} b(t) .
$$


Using an integration by parts formula, we see that $\left\{S^{*} \psi\right\}$ is an orthonormal set in $C_{a, b}^{\prime}[0, T]$ and

$$
\frac{1}{\sqrt{3}} b(T)^{3 / 2}\left(S^{*} \psi, x\right)^{\sim}=\left(S^{*} b, x\right)^{\sim}=\int_{0}^{T} x(t) d b(t) .
$$

Example 4.1. For given $\vec{m}=\left(m_{1}, \ldots, m_{n}\right) \in \mathbb{R}^{n}$ and $\overrightarrow{\sigma^{2}}=\left(\sigma_{1}^{2}, \ldots, \sigma_{n}^{2}\right) \in \mathbb{R}^{n}$ with $\sigma_{j}^{2}>0, j=1, \ldots, n$, let $\nu_{\vec{m}, \overrightarrow{\sigma^{2}}}$ be the Gaussian measure given by

$$
\nu_{\vec{m}, \overrightarrow{\sigma^{2}}}(B)=\left(\prod_{j=1}^{n} 2 \pi \sigma_{j}^{2}\right)^{-\frac{1}{2}} \int_{B} \exp \left\{-\sum_{j=1}^{n} \frac{\left(u_{j}-m_{j}\right)^{2}}{2 \sigma_{j}^{2}}\right\} d \vec{u}, \quad B \in \mathcal{B}\left(\mathbb{R}^{n}\right) .
$$

Then $\nu_{\vec{m}, \overrightarrow{\sigma^{2}}} \in \mathcal{M}\left(\mathbb{R}^{n}\right)$ and

$$
\widehat{\nu_{\vec{m}, \sigma^{2}}}(\vec{u})=\exp \left\{-\frac{1}{2} \sum_{j=1}^{n} \sigma_{j}^{2} u_{j}^{2}+i \sum_{j=1}^{n} m_{j} u_{j}\right\} .
$$

We can apply our results in previous section to the functionals of the form

$$
F_{1}(x)=\exp \left\{-\frac{1}{2} \sum_{j=1}^{n} \sigma_{j}^{2}\left[\left(g_{j}, x\right)^{\sim}\right]^{2}+i \sum_{j=1}^{n} m_{j}\left(g_{j}, x\right)^{\sim}\right\} .
$$

For example, in the case $n=1, g_{1}=S^{*} \psi, \vec{m}=m_{1}=0$ and $\overrightarrow{\sigma^{2}}=\sigma_{1}^{2}=$ $2 b(T)^{3} / 3$, we have

$$
F_{2}(x)=\exp \left\{-\left(\int_{0}^{T} x(t) d b(t)\right)^{2}\right\} .
$$

Using the equation (4.3), the Fubini theorem and the equation (2.2), we have that for each nonzero real number $q$,

$$
\begin{aligned}
& \int_{\mathbb{R}^{n}} \exp \left\{\frac{\|a\|_{C_{a, b}^{\prime}}}{\sqrt{|2 q|}} \sum_{j=1}^{n}\left|v_{j}\right|\right\}\left|d \nu_{\vec{m}, \overrightarrow{\sigma^{2}}}(\vec{v})\right| \\
& =\prod_{j=1}^{n}\left[\left(2 \pi \sigma_{j}^{2}\right)^{-1 / 2} \int_{-\infty}^{0} \exp \left\{-\frac{v_{j}^{2}}{2 \sigma_{j}^{2}}+\left(\frac{m_{j}}{\sigma_{j}^{2}}-\frac{\|a\|_{C_{a, b}^{\prime}}}{\sqrt{|2 q|}}\right) v_{j}-\frac{m_{j}^{2}}{2 \sigma_{j}^{2}}\right\} d v_{j}\right. \\
& \left.+\left(2 \pi \sigma_{j}^{2}\right)^{-1 / 2} \int_{0}^{+\infty} \exp \left\{-\frac{v_{j}^{2}}{2 \sigma_{j}^{2}}+\left(\frac{m_{j}}{\sigma_{j}^{2}}+\frac{\|a\|_{C_{a, b}^{\prime}}}{\sqrt{|2 q|}}\right) v_{j}-\frac{m_{j}^{2}}{2 \sigma_{j}^{2}}\right\} d v_{j}\right] \\
& <\prod_{j=1}^{n}\left[\left(2 \pi \sigma_{j}^{2}\right)^{-1 / 2} \int_{\mathbb{R}} \exp \left\{-\frac{v_{j}^{2}}{2 \sigma_{j}^{2}}+\left(\frac{m_{j}}{\sigma_{j}^{2}}-\frac{\|a\|_{C_{a, b}^{\prime}}}{\sqrt{|2 q|}}\right) v_{j}-\frac{m_{j}^{2}}{2 \sigma_{j}^{2}}\right\} d v_{j}\right. \\
& \left.+\left(2 \pi \sigma_{j}^{2}\right)^{-1 / 2} \int_{\mathbb{R}} \exp \left\{-\frac{v_{j}^{2}}{2 \sigma_{j}^{2}}+\left(\frac{m_{j}}{\sigma_{j}^{2}}+\frac{\|a\|_{C_{a, b}^{\prime}}}{\sqrt{|2 q|}}\right) v_{j}-\frac{m_{j}^{2}}{2 \sigma_{j}^{2}}\right\} d v_{j}\right] \\
& =\prod_{j=1}^{n}\left[\exp \left\{\frac{\sigma_{j}^{2}\|a\|_{C_{a, b}^{\prime}}^{2}}{2|2 q|}-\frac{m_{j}\|a\|_{C_{a, b}^{\prime}}}{\sqrt{|2 q|}}\right\}+\exp \left\{\frac{\sigma_{j}^{2}\|a\|_{C_{a, b}^{\prime}}^{2}}{2|2 q|}+\frac{m_{j}\|a\|_{C_{a, b}^{\prime}}}{\sqrt{|2 q|}}\right\}\right]
\end{aligned}
$$




$$
<+\infty
$$

Thus for all $q \in \mathbb{R}-\{0\}, E^{\operatorname{anf}_{q}}\left[F_{1}\right]$ (and so thus $E^{\operatorname{anf}_{q}}\left[F_{2}\right]$ ) exists and we can apply Theorems 3.6 and 3.7 to obtain the generalized Feynman integrals

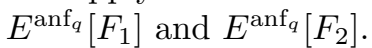

The functional

$$
F_{3}(x)=\exp \left\{i \int_{0}^{T} x(t) d b(s)\right\}
$$

also is a functional under our consideration because

$$
\begin{aligned}
F_{3}(x) & =\exp \left\{i\left(S^{*} b, x\right)^{\sim}\right\}=\exp \left\{\frac{i}{\sqrt{3}} b(T)^{3 / 2}\left(S^{*} \psi, x\right)^{\sim}\right\} \\
& =\int_{\mathbb{R}} \exp \left\{i\left(S^{*} \psi, x\right)^{\sim} v\right\} d \delta_{1}(v)=\widehat{\delta_{1}}\left(\left(S^{*} \psi, x\right)^{\sim}\right),
\end{aligned}
$$

where $\psi$ is given by the equation (4.1) and $\delta_{1}$ is the Dirac measure concentrated at $v=b(T)^{3 / 2} / \sqrt{3}$ in $\mathbb{R}$. Clearly, $\delta_{1}$ satisfies condition (3.10) for all $q \in \mathbb{R}-\{0\}$.

The functionals given by equations (4.4) and (4.5) arise naturally in quantum mechanics.

In next example we consider functionals of the form (3.1).

Example 4.2. Let $\tau: 0=t_{0}<t_{1}<\cdots<t_{m}=T$ be a partition of $[0, T]$.

(i) Let $h_{j}(t)=\int_{0}^{t} \chi_{\left[0, t_{j}\right]}(s) d b(s)$ for $j=1, \ldots, m$. Then $\left\{h_{1}, \ldots, h_{m}\right\}$ is a linearly independent subset of $C_{a, b}^{\prime}[0, T]$ and

$$
\begin{aligned}
F_{4}(x) & =\hat{\nu}\left(\left(h_{1}, x\right)^{\sim}, \ldots,\left(h_{m}, x\right)^{\sim}\right) \\
& =\hat{\nu}\left(x\left(t_{1}\right), \ldots, x\left(t_{m}\right)\right) .
\end{aligned}
$$

(ii) Let $h_{j}(t)=\int_{0}^{t} \chi_{\left[t_{j-1}, t_{j}\right]}(s) d b(s)$ for $j=1, \ldots, m$. Then $\left\{h_{1}, \ldots, h_{m}\right\}$ is an orthogonal subset of $C_{a, b}^{\prime}[0, T]$ and

$$
\begin{aligned}
F_{5}(x) & =\hat{\nu}\left(\left(h_{1}, x\right)^{\sim}, \ldots,\left(h_{m}, x\right)^{\sim}\right) \\
& =\hat{\nu}\left(x\left(t_{1}\right), x\left(t_{2}\right)-x\left(t_{1}\right), \ldots, x\left(t_{m}\right)-x\left(t_{m-1}\right)\right) .
\end{aligned}
$$

(iii) For each $j=1, \ldots, m$, let $w_{j}(t)=\int_{0}^{t} \chi_{\left[0, t_{j}\right]}(s) d b(s)$. Then $\left\{w_{1}, \ldots, w_{m}\right\}$ is a linearly independent subset of $C_{a, b}^{\prime}[0, T]$. Let $h_{j}(t)=S^{*} w_{j}(t)$ for each $j=1, \ldots, m$. Since the linear operator $S$ is injective, $\left\{h_{1}, \ldots, h_{m}\right\}$ also is linearly independent. Using an integration by parts formula, we see that

$$
\left(h_{j}, x\right)^{\sim}=\int_{0}^{t_{j}} x(t) d b(t)
$$

for each $j=1, \ldots, m$. In this case,

$$
\begin{aligned}
F_{6}(x) & =\hat{\nu}\left(\left(h_{1}, x\right)^{\sim}, \ldots,\left(h_{m}, x\right)^{\sim}\right) \\
& =\hat{\nu}\left(\int_{0}^{t_{1}} x(s) d b(s), \ldots, \int_{0}^{t_{m}} x(s) d b(s)\right) .
\end{aligned}
$$


In $[1,4,5]$, the functionals of the forms $(4.6),(4.7)$ and (4.8) were studied on classical Wiener space. By using the Gram-Schmidt process, the functionals $F_{k}(k=4,5,6)$ can be rewritten in the form the equation (3.2) for suitable orthonormal subset $\left\{g_{1}, \ldots, g_{n}\right\}$ of $C_{a, b}^{\prime}[0, T]$, respectively.

Based on quantum mechanics considerations, the most important value of the parameter $q$ in $E^{\operatorname{anf}_{q}}[F]$ is $q=-1$. Thus, if the complex measure $\nu$ corresponding $F_{k}$ satisfies condition (3.10) with $q$ replaced with $q_{0} \in(0,1)$ as the measures $\nu_{\vec{m}, \overrightarrow{\sigma^{2}}}$ and $\delta_{1}$ in Example 4.1 , we can obtain the generalized analytic Feynman integrals $E^{\operatorname{anf}_{-1}}\left[F_{k}\right](k=4,5,6)$ by using the equation (3.11).

\section{References}

[1] R. H. Cameron and D. A. Storvick, An $L_{2}$ analytic Fourier-Feynman transform, Michigan Math. J. 23 (1976), no. 1, 1-30.

[2] _ Relationships between the Wiener integral and the analytic Feynman integral, Rend. Circ. Mat. Palermo (2) Suppl. No. 17 (1987), 117-133.

[3] Change of scale formulas for Wiener integral, Rend. Circ. Mat. Palermo (2) Suppl. No. 17 (1987), 105-115.

[4] K. S. Chang, G. W. Johnson, and D. L. Skoug, Necessary and sufficient conditions for the Fresnel integrability of certain classes of functions, J. Korean Math. Soc. 21 (1984), no. 1, 21-29.

[5] - Necessary and sufficient conditions for membership in the Banach algebra $S$ for certain classes of functions, Rend. Circ. Mat. Palermo (2) Suppl. No. 17 (1987), $153-171$.

[6] S. J. Chang and D. M. Chung, Conditional function space integrals with applications, Rocky Mountain J. Math. 26 (1996), no. 1, 37-62.

[7] S. J. Chang, J. G. Choi, and D. Skoug, Integration by parts formulas involving generalized Fourier-Feynman transforms on function space, Trans. Amer. Math. Soc. 355 (2003), no. 7, 2925-2948.

[8] Evaluation formulas for conditional function space integrals. I, Stoch. Anal. Appl. 25 (2007), no. 1, 141-168.

[9] S. J. Chang and D. Skoug, Generalized Fourier-Feynman transforms and a first variation on function space, Integral Transforms Spec. Funct. 14 (2003), no. 5, 375-393.

[10] B. S. Kim and T. S. Kim, Change of scale formulas for Wiener integral over paths in abstract Wiener space, Commun. Korean Math. Soc. 21 (2006), no. 1, 75-88.

[11] Y. S. Kim, Analytic Feynman integrals, Fourier-Feynman transforms and change of scale formula for Wiener integrals over paths on abstract Wiener spaces, Integral Transforms Spec. Funct. 16 (2005), no. 4, 323-335.

[12] Y. S. Kim, J. M. Ahn, K. S. Chang, and I. Yoo, A change of scale formula for Wiener integrals on the product abstract Wiener spaces, J. Korean Math. Soc. 33 (1996), no. 2, 269-282.

[13] H. L. Royden, Real Analysis, Third edition, Macmillan, 1988.

[14] J. Yeh, Singularity of Gaussian measures on function spaces induced by Brownian motion processes with non-stationary increments, Illinois J. Math. 15 (1971), 37-46.

[15] $\ldots$ Stochastic Processes and the Wiener Integral, Marcel Dekker, Inc., New York, 1973.

[16] I. Yoo, Sequential Yeh-Feynman integrals, Doctoral Thesis, Yonsei University, 1987.

[17] I. Yoo and D. Skoug, A change of scale formula for Wiener integrals on abstract Wiener spaces, Internat. J. Math. Math. Sci. 17 (1994), no. 2, 239-247.

[18] _ A change of scale formula for Wiener integrals on abstract Wiener spaces. II, J. Korean Math. Soc. 31 (1994), no. 1, 115-129. 
[19] I. Yoo, T. S. Song, and B. S. Kim, A change of scale formula for Wiener integrals of unbounded functions. II, Commun. Korean Math. Soc. 21 (2006), no. 1, 117-133.

[20] I. Yoo, T. S. Song, B. S. Kim, and K. S. Chang, A change of scale formula for Wiener integrals of unbounded functions, Rocky Mountain J. Math. 34 (2004), no. 1, 371-389.

[21] I. Yoo and G. J. Yoon, Change of scale formulas for Yeh-Wiener integrals, Commun. Korean Math. Soc. 6 (1991), no. 1, 19-26.

Seung Jun Chang

Department of Mathematics

DANKOOK UNIVERSITY

Cheonan 330-714, Korea

E-mail address: sejchang@dankook.ac.kr

Jae Gil Choi

Department of Mathematics

DANKOOK UNIVERSITY

Cheonan 330-714, Korea

E-mail address: jgchoi@dankook.ac.kr

Hyun Soo Chung

Department of Mathematics

DANKOOK UNIVERSITY

Cheonan 330-714, Korea

E-mail address: hschung@dankook.ac.kr 